\title{
Neutrino phenomenology from leptogenesis
}

\author{
Stefano Morisi* \\ INFN - Sezione di Napoli, Complesso Univ. Monte S. Angelo, I-80126 Napoli, Italy and \\ Dipartimento di Fisica "Ettore Pancini", Università degli studi di Napoli "Federico II", \\ Complesso Univ. Monte S. Angelo, I-80126 Napoli, Italy \\ E-mail: morisi@na.infn.it
}

\begin{abstract}
We consider an extension of the Standard Model with three heavy right-handed neutrino. Active neutrino get a mass with the seesaw mechanism and baryon asymmetry is generated through leptogenesis scenario. Moreover we consider a reasonable hierarchical structure for Dirac neutrino mass matrix, similar to up-type quark mass matrix. Under such a conditions it follows a relevant connection between high scale CP violation and low energy Dirac neutrino CP phase. Leptogenesis requires a compact heavy right-handed neutrino mass spectrum that implies a relation between low energy neutrino parameters. In particular we obtain that the Dirac CP phase is restricted in the range $-0.90 \pi<\delta<-0.75 \pi$ and the lightest active neutrino mass in the range $m_{1} \sim(0.002-0.004) \mathrm{eV}$.
\end{abstract}

Neutrino Oscillation Workshop (NOW2018)

9 - 16 September, 2018

Rosa Marina (Ostuni, Brindisi, Italy)

${ }^{*}$ Speaker. 


\section{Introduction}

The measure of neutrino square mass differences require to extension of the electroweak Standard Model. We consider a model with three heavy right-handed Standard Model singlets $N_{i}$. The three active light neutrinos get a Majorana mass from the type-I seesaw mechanism. In this framework the $\mathrm{CP}$ violating out of equilibrium decay of $N_{i}$ can produce a lepton asymmetry that is then converted into a baryon asymmetry (baryogenesis via leptogenesis [1]). The possible correlations between the high energy scale CP violation and the low energy one has been intensively studied in literature $[2,3,4]$. The existence of such kind of relation can be naively understood by inverting the seesaw relation, namely

$$
M_{R}=-M_{D}^{T} M_{v}^{-1} M_{D}
$$

The lepton asymmetry generated by leptogenesis depends on the right handed neutrino couplings and their masses, namely by the matrices $M_{R}$ and $M_{D}$. By fixing the Dirac neutrino mass matrix $M_{D}$, one gets from Eq. (1.1) a connection between light neutrino mass matrix $M_{v}$ and lepton asymmetry that is related to the high energy scale CP violation. Unfortunately the experiments do not provide direct information about the Dirac mass matrix, and therefore in order to fix $M_{D}$ we have to evoke some theoretical arguments as also given for example in [2], [3] and [4]. A possibility to fix the structure of the Dirac mass matrix is to assume a Grand Unified gauge group like $S O(10)$ that implies $M_{D} \approx M_{\text {up }}$, where $M_{\text {up }}$ denotes the up-type quark mass matrix. Actually the unrealistic $S O(10)$ model with only one 10-Higgs scalar field predicts exactly $M_{D}=M_{\text {up }}$. In this case we get $Y_{11}^{v} \ll Y_{22}^{v} \ll Y_{33}^{v}$ and therefore it follows that also the right-handed neutrino mass matrix results to be hierarchical unless the entries of the matrix $M_{v}$ are strongly constrained and we have $M_{R_{1}} \ll$ $M_{R_{2}} \ll M_{R_{3}}$. Such a hierarchy could give problem in order to obtain the correct BAU. Indeed, in case the heaviest of the right-handed neutrinos has a mass of about $M_{R_{3}} \approx 10^{14} \mathrm{GeV}$ (that is around the grand unified scale) the lightest right-handed mass could be below the Davidson-Ibarra limit $M_{R_{1}}<10^{9} \mathrm{GeV}$ [5], which is the lower limit for a lepton asymmetry generated by the decay of the lightest right-handed neutrino to be sufficiently large as shown also in [3]. A mechanism to obtain a reasonable value for the lepton asymmetry in this context has been proposed in [6]. The idea is quite simple: by imposing a compact spectrum for the right-handed masses on the 1.h.s. of Eq. (1.1), it follows a condition for neutrino mass parameters on the r.h.s. of Eq. (1.1) (see also [3]) once the structure of the Dirac neutrino mass matrix $M_{D}$ is fixed. If $V_{L, R}$ are unitary matrices that diagonalize $M_{D}$ we have

$$
M_{R}=-V_{R}^{T} M_{D}^{\mathrm{diag}} A M_{D}^{\mathrm{diag}} V_{R} ; \quad A=V_{L}^{*} U_{\mathrm{PMNS}}\left(M_{v}^{\mathrm{diag}}\right)^{-1} U_{\mathrm{PMNS}}^{T} V_{L}^{\dagger}
$$

where $M_{D}^{\text {diag }}=\left(M_{D_{1}}, M_{D_{2}}, M_{D_{3}}\right)$ and $U_{\mathrm{PMNS}}$ is the neutrino mixing matrix and $A$ is a funcion of the two neutrino square mass differences $\Delta m_{21}^{2}$ and $\Delta m_{31}^{2}$, the mixing angles $\theta_{12}, \theta_{23}$ and $\theta_{13}$, the Dirac $\mathrm{CP} \delta$ and the Majorana phases $\alpha, \beta$. Once the matrices $V_{L, R}$ and $M_{D}^{\text {diag }}$ are fixed, then a compact $M_{R}$ spectrum follows if [6]

$$
A_{23}=A_{33}=0 .
$$

Under these assumptions two of the three eigenvalues of the right-handed neutrino mass matrix are degenerate $M_{R_{2}}=M_{R_{3}}=\left|A_{13}\right| M_{D_{1}} M_{D_{3}}$ and $M_{R_{1}}=\left|A_{22}\right| M_{D_{2}}^{2}$. In particular within $S O(10)$ framework we expect $M_{D} \approx M_{\text {up }}, M_{\ell} \approx M_{\text {down }}$. Assuming the down quark $M_{\text {down }}$ and charged lepton 
$M_{\ell}$ mass matrices approximatively diagonal, it follows that the up quark mass matrix must be diagonalized by the CKM mixing matrix. Moreover if the scalar sector of the $S O(10)$ model does not contain the 120 irreducible representation, then both $m_{D}$ and $M^{\text {up }}$ are symmetric and $M_{D} \approx V_{\mathrm{CKM}}^{\dagger} M_{\mathrm{up}}^{\mathrm{diag}} V_{\mathrm{CKM}}^{*}$ where $M_{\mathrm{up}}^{\mathrm{diag}} \equiv\left\{m_{u}, m_{c}, m_{t}\right\}$ that are the physical up, charm and top quark masses and $V_{L} \equiv V_{\mathrm{CKM}}$.

The two positions $A_{23}=A_{33}=0$ are complex equations that can be used to predict four neutrino mixing parameters from the other ones. Since the parameters $m_{1}, \delta, \alpha$, and $\beta$ are still experimentally undetermined the better choice is to use $\Delta m_{21}^{2}, \Delta m_{31}^{2}, \theta_{12}, \theta_{23}, \theta_{13}$ to obtain unknown parameters. The current bounds on neutrino mixing parameters can be found for example in Ref. [7]. We randomly extract 10000 points and for each input random point we get a set of output values for the lightest neutrino mass and the three neutrino phases. The results are given in Figure 1 where we show the mass $m_{1}$ as a function of the Dirac phase $\delta$ (left panel) and the correlation between the Majorana phases $\alpha$ and $\beta$ (right panel). In the left plot we report the $1 \sigma$ (dot-dashed lines) and the $3 \sigma$ (dashed lines) experimental range for the Dirac phase, while the dashed red line is the best fit value of the Dirac phase. We note that under the above hypotheses (see Eq. 1.3) a link between the lightest neutrino mass and the Dirac phase is expected. The scatter plot of Figure 1 (grey points in left panel) shows such correlation when the uncertainty on the input parameters is taken into account. Despite the high number of 10000 generated points, only a small part of these (about 30 circled in red in Figure) led to a value for the baryon asymmetry consistent with the experimental value $Y_{\Delta B}=(8.65 \pm 0.06) \cdot 10^{-11}$. We notice that one of these regions falls outside the $3 \sigma$ confidence range for $\delta$. Moreover, the lightest neutrino mass has an upper and lower limit
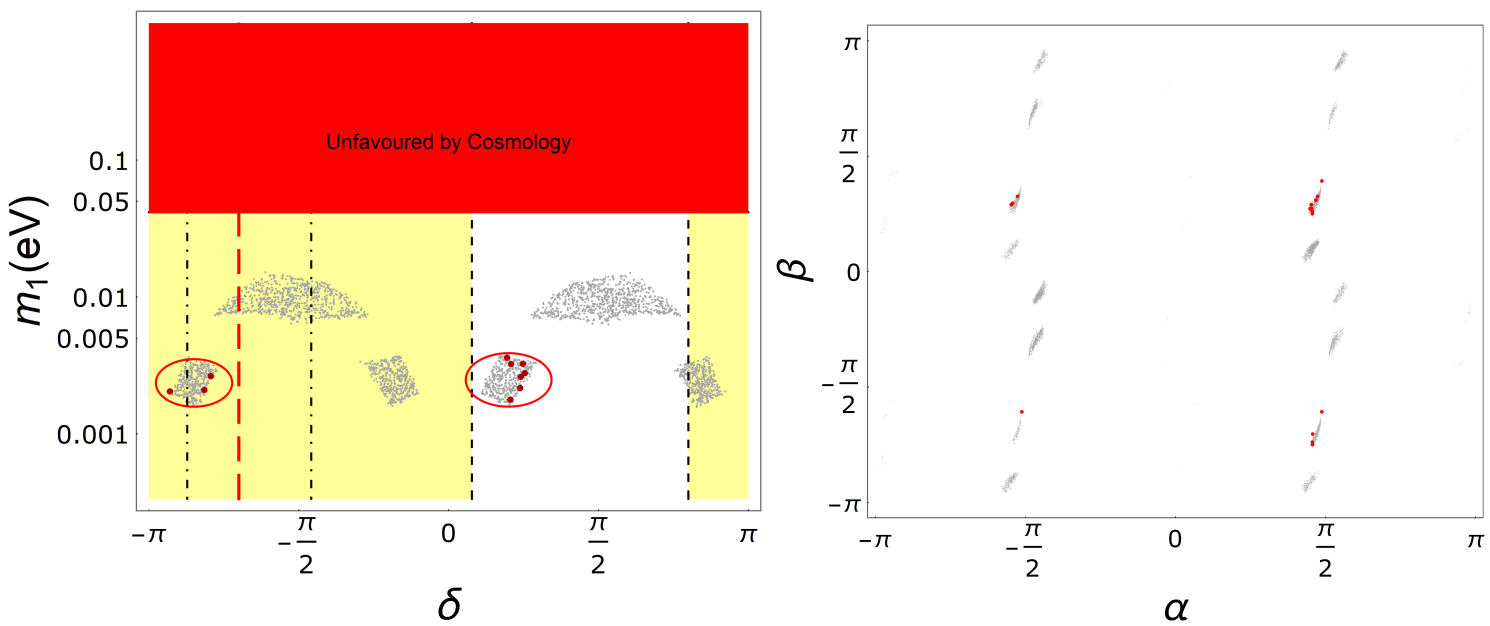

Figure 1: (Left panel) Lightest neutrino mass vs the Dirac phase. The different vertical bands correspond to the experimental values for the Dirac phase. In yellow the $3 \sigma$ confidence band is evidenced. (Right panel) Majorana phases $\alpha$ and $\beta$ for the numerically generated points. In both plots red points are obtained by imposing $Y_{\Delta B}$ within the 3- $\sigma$ experimental range while grey points are not constrained from baryon asymmetry.

that is not affected by present cosmological observations; the regions efficient for leptogenesis, in fact, have $m_{1} \sim(0.002,0.004) \mathrm{eV}$ and $\delta \sim(-0.90 \pi,-0.75 \pi)$. Also the Majorana phases are constrained, in particular $|\alpha| \approx \pi / 2$ and $\beta$ is distributed in the range $(-\pi,+\pi)$ in two isolated 
regions centered around the values of $0.3 \pi$ and $-0.7 \pi$. The neutrinoless double beta parameter $m_{\beta \beta}=\left|\sum_{i} m_{i} U_{P M N S_{e i}}^{2}\right|$ is found to lie between $0.001 \mathrm{eV}$ and $0.02 \mathrm{eV}$, which is below the experimental bounds, set, for example, in [8]. In Figure 2 we give the Jarlskog parameter [9] as a function of the baryon asymmetry yield, together with the red vertical band representing the $3 \sigma$ confidence range for the baryon abundance experimentally measured. We see that requiring a baryon asymmetry within about a $3-\sigma$ range around the experimental value of the baryon abundance, we get $J$ in the range $(-0.022,-0.018)$.

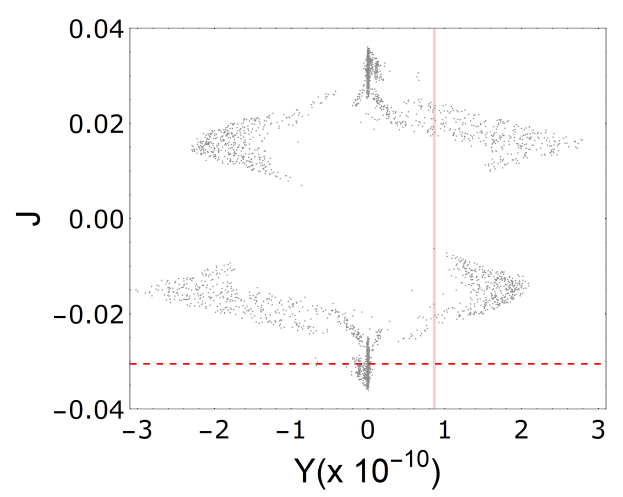

Figure 2: Jarlskog invariant parameter as a function of the baryon asymmetry yield obtained. The vertical band correspond to the $3-\sigma$ experimental value The horizontal dashed line represents the value obtained by fixing all the oscillating parameters to their best fit values.

\section{References}

[1] M. Fukugita and T. Yanagida, Phys. Lett. B 174, 45 (1986). doi:10.1016/0370-2693(86)91126-3

[2] M. S. Berger and B. Brahmachari, Phys. Rev. D 60, 073009 (1999) doi:10.1103/PhysRevD.60.073009 [hep-ph/9903406].

[3] E. K. Akhmedov, M. Frigerio and A. Y. Smirnov, JHEP 0309, 021 (2003) doi:10.1088/1126-6708/2003/09/021 [hep-ph/0305322].

[4] P. H. Frampton, S. L. Glashow and T. Yanagida, Phys. Lett. B 548, 119 (2002) doi:10.1016/S0370-2693(02)02853-8 [hep-ph/0208157].

[5] S. Davidson and A. Ibarra, Phys. Lett. B 535, 25 (2002) doi:10.1016/S0370-2693(02)01735-5 [hep-ph/0202239].

[6] F. Buccella, D. Falcone, C. S. Fong, E. Nardi and G. Ricciardi, Phys. Rev. D 86, 035012 (2012) doi:10.1103/PhysRevD.86.035012 [arXiv:1203.0829 [hep-ph]].

[7] F. Capozzi, E. Di Valentino, E. Lisi, A. Marrone, A. Melchiorri and A. Palazzo, Phys. Rev. D 95, no. 9, 096014 (2017) doi:10.1103/PhysRevD.95.096014 [arXiv:1703.04471 [hep-ph]].

[8] M. Agostini et al. [GERDA Collaboration], Phys. Rev. Lett. 120, no. 13, 132503 (2018) doi:10.1103/PhysRevLett.120.132503 [arXiv:1803.11100 [nucl-ex]].

[9] C. Jarlskog, Phys. Rev. Lett. 55, 1039 (1985). doi:10.1103/PhysRevLett.55.1039 